\title{
Fatores determinantes e a incidência de tuberculose na população indígena: Uma revisão integrativa da literatura
}

The incidence of tuberculosis in the indigenous population and its determining factors: An integrative literature review

Factores determinantes y la incidencia de tuberculosis en la población indígena: Una revisión integradora de la literatura

Elian Coimbra Fontinelli Tavares ORICID: https://orcid.org/0000-0003-2886-935X Universidade Federal do Pará, Brasil E-mail: judacoimbra@gmail.com Leonardo Carvalho da Silva ORCID: https://orcid.org/0000-0001-8554-2391 Universidade Federal do Pará, Brasil E-mail: leonardohab2020@gmail.com

Susany dos Santos Tenório ORCID: https://orcid.org/0000-0003-1849-2480 Universidade Federal do Pará, Brasil E-mail: susanystenorio@gmail.com Daniely da Silva Sena ORCID: https://orcid.org/0000-0003-3571-8293 Universidade Federal do Pará, Brasil E-mail: sennadaniely@gmail.com Gabrielli Batista de Alencar ORCID: https://orcid.org/0000-0003-0984-6275 Universidade Federal do Pará, Brasil E-mail: alencargabrielli@gmail.com

Thais Karoline Santiago Ribeiro ORCID: https://orcid.org/0000-0002-4622-4371 Universidade Federal do Pará, Brasil E-mail: thais.ribeiro@ics.ufpa.br

Maria Eduarda Libório Martins ORCID: https://orcid.org/0000-0002-2217-4103 Universidade Federal do Pará, Brasil E-mail: mliboriomartins@gmail.com

Thamyles da Silva Dias ORCID: https://orcid.org/0000-0002-8797-9025 Universidade Federal do Pará, Brasil E-mail: thamyles.dias@gmail.com

Douglas do Nascimento Galvão ORCID: https://orcid.org/0000-0001-5277-0718 Universidade Federal do Pará, Brasil E-mail: douglasnascimentogalvao@gmail.com

Juliana Marques de Moraes Carneiro ORCID: https://orcid.org/0000-0003-0611-6154 Universidade Federal do Pará, Brasil E-mail: julianacarneiro134@gmail.com

Letícia Cristina Bastos De Sousa ORCID: https://orcid.org/0000-0003-1708-1380 Universidade Federal do Pará, Brasil E-mail: letcrist64@gmail.com

Bárbara Letícia Corrêa Gomes ORCID: https://orcid.org/0000-0002-9875-8981 Universidade Federal do Pará, Brasil E-mail: barbaraltc4gomes@gmail.com Arthur Filocreão dos Santos Oliveira ORCID: https://orcid.org/0000-0002-8413-9581 Universidade Federal do Pará, Brasil 


\author{
Carmem Irany dos Santos Oliveira \\ ORCID: https://orcid.org/0000-0001-8997-9806 \\ Universidade Federal do Pará, Brasil \\ E-mail: irany2010@gmail.com \\ Evanildo Lopes Monteiro \\ ORCID: https://orcid.org/ 0000-0003-3501-6267 \\ Universidade Federal do Pará, Brasil \\ E-mail: emonteiro@ufpa.br
}

\begin{abstract}
Resumo
O presente estudo tem como objetivo identificar os fatores determinantes da ocorrência de tuberculose na população indígena, mapear a incidência dessa doença dentre o período de 2015 a 2019 na região Norte e contribuir para o enfrentamento dessa problemática. Realizou-se um estudo descritivo, do tipo revisão integrativa de literatura (RIL), com enfoque em artigos científicos destinados a resumir evidências sobre os fatores determinantes para o surgimento e prevalência de tuberculose nas populações indígenas. Foram selecionados 9 artigos publicados em periódicos entre o período de 2016 a 2021 nos seguintes bancos de dados bibliográficos: Biblioteca Virtual em Saúde (BVS) e PubMed. Dessa forma, 6 artigos (66,7\%) foram encontrados na base de dados da Biblioteca Virtual em Saúde (BVS) e 3 (33,3\%) na PubMed. A ampla maioria dos trabalhos aborda como fatores determinantes a descontinuidade do tratamento, as questões socioeconômicas, a dificultosa acessibilidade às unidades de saúde e a imprecisão diagnóstica. A vivência de situações de exclusão, invisibilidade e discriminação colocam as comunidades indígenas em situações de vulnerabilidade. A superação dos fatores determinantes para a prevalência de tuberculose nessas populações envolve questões multissetoriais. Políticas Públicas, distribuição de renda, acessibilidade aos sistemas de saúde e a implementação do PNCT são alguns dos pontos essenciais para a diminuição dos casos.
\end{abstract}

Palavras-chave: Indígena; Tuberculose; Vulnerabilidade.

\begin{abstract}
The present study aims to identify the factors behind the occurrence of tuberculosis in the indigenous population; map this disease between 2015 to 2019, in the northern region of Brazil, and contribute to addressing this problem. A descriptive study was carried out, of the type integrative literature review (RIL), focusing on specialized articles, review of evidence on the determining factors for the emergence and prevalence of tuberculosis in indigenous populations. Nine articles published in journals between 2016 and 2021 were selected in the following bibliographic databases: Biblioteca Virtual em Saúde (BVS) and PubMed. Thus, 6 articles $(66.7 \%)$ were found in the VHL database and 3 $(33.3 \%)$ in PubMed. Most of the studies deal with determining factors for discontinuing treatment, socioeconomic issues, difficult accessibility - such as health units and diagnostic inaccuracy. In addition, there are reports of exclusion, invisibility and discrimination. All of this puts indigenous communities in situations of vulnerability. Overcoming the factors that make tuberculosis prevalence feasible in these categories involves multisectoral issues: public policies, income distribution, accessibility to health systems and the implementation of the PNCT are some of the essential points for reducing cases.
\end{abstract}

Keywords: Indigenous; Tuberculosis; Vulnerability.

\title{
Resumen
}

El presente estudio tiene como objetivo identificar los factores determinantes de la ocurrencia de tuberculosis en la población indígena, mapear la incidencia de esta enfermedad entre el período de 2015 a 2019 en la región Norte y contribuir a enfrentar esta problemática. Se realizó un estudio descriptivo, como un tipo de revisión bibliográfica integradora (RL), centrado en artículos científicos destinados a resumir evidencias sobre los determinantes de la aparición y prevalencia de la tuberculosis en poblaciones indígenas. Se seleccionaron 9 artículos publicados en revistas entre el periodo de 2016 a 2021 en las siguientes bases de datos bibliográficas: Biblioteca Virtual de Salud (BVS) y PubMed. Resultados: Se seleccionaron 6 artículos (66,7\%) en la base de datos de la Biblioteca Virtual de Salud (BVS) y $3(33,3 \%)$ en PubMed. La mayoría de los trabajos abordan como factores determinantes el descontinuidad del tratamiento, las cuestiones socioeconómicas, la difículdad de accesibilidad a los establecimientos de de salud y la imprecisión del diagnóstico. La vivencia de situaciones de exclusión, invisibilidad y discriminación colocan a las comunidades indígenas en situaciones de vulnerabilidad. Superar los factores determinantes de la prevalencia de la tuberculosis en astas poblaciones implica cuestiones multisectoriales. Las políticas públicas, la distribución del ingresos, la accesibilidad a los sistemas sanitarios y la aplicación del PNCT son algunos de los puntos esenciales para la reducción de los casos.

Palabras clave: Indígena; Tuberculosis; Vulnerabilidad.

\section{Introdução}

A desigualdade e o preconceito vivenciado frequentemente pelos povos indígenas estão ligados ao processo de ocupação e colonização do território brasileiro. Esse momento histórico que dizimou etnias e alterou significativamente o modo de vida 
dos que sobreviveram também deixou um legado de marginalização social, o qual se manifesta sobretudo no âmbito da saúde. Assim, a discriminação e a condição de vida precária favorecem o surgimento de diversas doenças que afetam ainda mais o bemestar físico, psíquico e social desses povos. (Sandes, Freitas, Souza \& Leite, 2018; Souza, 2017).

Nesse contexto, a tuberculose (TB) - doença infecciosa causada pelo Micobacterium tuberculosis que atinge os pulmões, e está relacionada às condições socioeconômicas e ambientais - vem se destacando consideravelmente em decorrência da sua maior incidência nas populações indígenas do que na população não indígena (Paiva et al., 2019).

Corroborando a severidade deste cenário, o Programa Nacional de Controle da Tuberculose (PNCT) afirma que a doença constitui um sério problema de saúde pública, uma vez que está intimamente associada à pobreza e à má distribuição de renda. Concomitante a isso, a população indígena se torna mais suscetível para a TB, pois pertence a um grupo de vulnerabilidade tanto econômica quanto social (Brasil, 2004; Castro et al., 2018).

Diante disso, em prol do processo de inclusão aos serviços de saúde, instituiu-se a Política Nacional de Atenção à Saúde dos Povos Indígenas (PNASPI), a qual preconiza o propósito de "garantir aos povos indígenas o acesso à atenção integral à saúde, contemplando a diversidade social, cultural, geográfica, histórica e política de modo a favorecer a superação dos fatores que tornam essa população mais vulnerável aos agravos à saúde" (Brasil, 2002).

Ademais, é importante ressaltar a incompletude de dados do perfil epidemiológico dessa população, devido à precariedade do sistema de registro de informações, o qual é evidenciado pela subnotificação que ocorre por meio de falhas no preenchimento do campo raça/cor nas fichas de notificação e pelas características geográficas (Paiva et al., 2017; Ferreira, Santos, Oliveira \& Caldas, 2020). Por conseguinte, os casos não informados poderiam ser utilizados para avaliar a gravidade da endemia, atraso na detecção de casos, se houve início tardio do tratamento e a sua efetividade. Além disso, poderiam destacar as intercorrências no acesso ao diagnóstico em tempo oportuno e avaliar a qualidade do serviço de saúde nessas áreas. (Malacarne, 2013)

Apesar disso, em 2017 foram notificados 69.569 casos novos de TB no Brasil, que corresponde a um coeficiente de incidência igual a 33,5 casos/100 mil habitantes, já na região Norte o coeficiente foi maior que a média nacional, com 42,7 casos novos/100 mil habitantes. Em relação aos povos indígenas, a incidência de TB é ainda maior em comparação a população não indígena (Paiva et al., 2019).

A partir disso, observa-se o desamparo na saúde enfrentado pelos povos indígenas, tornando-os mais suscetíveis às diversas doenças, principalmente à TB. Tal contexto é resultado de vários elementos como: a situação socioeconômica, alto índice de desnutrição e baixa escolaridade. As condições sanitárias são evidenciadas pela falta de saneamento básico, precárias condições habitacionais e o padrão de transmissão nas aldeias. Também, há de se ressaltar os fatores culturais que interferem no atendimento, como a carência de intérpretes culturais que facilitam a troca de informações entre o paciente e o profissional responsável e a valorização da medicina tradicional desses povos. Além disso, o difícil acesso à saúde e a baixa cobertura da atenção primária são determinantes que colaboram para a perpetuação da doença na sociedade indígena. (Gomes \& Esperidião, 2017; Zorzi, 2019; Souza, 2017).

Desse modo, o objetivo deste estudo é elucidar - por meio de artigos científicos - os fatores socioculturais, estruturais, geográficos que vulnerabilizam a população estudada à TB e como tais fatores contribuem para a alta prevalência de tuberculose entre as comunidades e aldeias indígenas da região Norte.

\section{Metodologia}

Realizou-se um estudo descritivo, do tipo revisão integrativa de literatura (RIL) com enfoque em artigos científicos destinados a reunir evidências sobre os fatores determinantes para o surgimento e prevalência de tuberculose nas populações indígenas. A investigação, por este método, percorre seis etapas distintas: identificação do tema e definição da questão de 
pesquisa; estabelecimento de critérios para inclusão e exclusão de estudos; definição das informações a serem extraídas dos estudos; interpretação dos resultados e apresentação da síntese dos dados (Ercole, Melo \& Alcoforado, 2014; Crossetti, 2012).

Como estratégia de busca, empregaram-se descritores previamente submetidos aos índices de descritores da DeCS (Descritores em Ciências da Saúde): Indígenas; Tuberculose; Vulnerabilidade.

A questão de pesquisa envolve: "Quais as evidências científicas sobre a frequência de casos de tuberculose entre povos indígenas, no período de 2015 a 2019 e quais os fatores determinantes para a incidência da doença na referida população?’. Para essa revisão integrativa foram adotados como critérios de inclusão: artigos publicados em Língua Portuguesa, compreendidos entre o período de 2016 a 2021 e que sejam compatíveis com a temática e os objetivos do presente trabalho. Considerou-se os critérios de exclusão: artigos publicados em língua estrangeira; monografias; artigos incompletos e com mais de 5 anos da data de publicação.

A análise dos estudos relevantes deu-se por meio da análise temática da literatura, cujo objetivo envolve a detecção de padrões, regularidades e inconsistências a fim da busca de evidências, fatores e dados para identificação de temas relevantes (Polit \& Beck, 2019). No presente estudo, a detecção dos fatores determinantes para o aparecimento de tuberculose nas populações indígenas, os dados epidemiológicos envolvendo a incidência e prevalência de tuberculose e o papel desempenhado pelo profissional da enfermagem na resolução desses casos foram questões norteadoras para análise da literatura.

Foram selecionados 9 artigos publicados em periódicos entre o período de 2016 a 2021 nos seguintes bancos de dados bibliográficos: Biblioteca Virtual em Saúde (BVS) e PubMed.

\section{Resultados}

O levantamento do material bibliográfico para a construção deste trabalho contou com a análise e seleção de 10 artigos. Destes, 6 (66,7\%) foram encontrados na base de dados da Biblioteca Virtual em Saúde (BVS) e 3 (33,3\%) na PubMed.

A organização deste artigo foi realizada por meio da realização de codificação, na qual A se refere aos artigos que vão de A1 a A9 dispostos no quadro abaixo.

A quantidade de autores participantes dos artigos soma um quantitativo de 41. Destes, 24 (58,4\%) são enfermeiros, 7 (17,1\%) são médicos, 3 (7,3\%) são biólogos, 2 (4,7\%) são historiadores, 1 (2,5\%) é farmacêutico, 1 (2,5\%) é psicólogo, 1 (2,5\%) é bioquímico, 1 (2,5\%) é assistente social e 1 (2,5\%) é estatístico. A participação de autores fora da área da saúde demonstra que este assunto é amplo e de abrangência intersetorial. 
Quadro 1: Caracterização do material bibliográfico.

\begin{tabular}{|c|c|c|c|c|c|c|}
\hline Cód. & Título & $\begin{array}{r}\text { Quantidade } \\
\text { de Autores }\end{array}$ & $\begin{array}{c}\text { Ano de } \\
\text { Publicação }\end{array}$ & Área de Publicação & Fonte & $\begin{array}{c}\text { Abordagem/ Tipo de } \\
\text { Estudo }\end{array}$ \\
\hline A.1 & $\begin{array}{l}\text { Saúde Indígena: Reflexões } \\
\text { Contemporâneas }\end{array}$ & 1 & 2018 & História & BVS & $\begin{array}{l}\text { Qualitativo/ Bibliográfico- } \\
\text { interpretativo }\end{array}$ \\
\hline A.2 & $\begin{array}{c}\text { Tuberculose entre crianças e } \\
\text { adolescentes indígenas no Brasil: fatores } \\
\text { associados ao óbito e ao abandono do } \\
\text { tratamento }\end{array}$ & 4 & 2019 & $\begin{array}{l}\text { Enfermagem } \\
\text { Medicina }\end{array}$ & PubMed & Quantitativo/ Documental \\
\hline A.3 & $\begin{array}{l}\text { Tendência da tuberculose em indígenas } \\
\text { no Brasil no período de 2011-2017 }\end{array}$ & 4 & 2020 & $\begin{array}{l}\text { Enfermagem } \\
\text { Estatística }\end{array}$ & BVS & $\begin{array}{l}\text { Quantitativo- ecológico/ } \\
\text { Documental }\end{array}$ \\
\hline A.4 & $\begin{array}{c}\text { Modelo preditivo de determinantes } \\
\text { socioeconômicos da tuberculose em } \\
\text { população indígena do estado do Pará, } \\
\text { brasil }\end{array}$ & 6 & 2019 & $\begin{array}{c}\text { Enfermagem } \\
\text { Medicina }\end{array}$ & BVS & $\begin{array}{c}\text { Estudo analítico- } \\
\text { quantitativo/ Bibliográfico }\end{array}$ \\
\hline A.5 & $\begin{array}{l}\text { Distribuição espacial de tuberculose nas } \\
\text { populações indígenas e não indígenas do } \\
\text { estado do Pará, Brasil, 2005-2013 }\end{array}$ & 6 & 2017 & Enfermagem & BVS & $\begin{array}{l}\text { Quantitativo- ecológico/ } \\
\text { Documental }\end{array}$ \\
\hline A.6 & $\begin{array}{l}\text { Acesso dos usuários indígenas aos } \\
\text { serviços de saúde de Cuiabá, Mato } \\
\text { Grosso, Brasil. }\end{array}$ & 2 & 2017 & $\begin{array}{l}\text { Serviço social } \\
\text { Psicologia }\end{array}$ & PubMed & Qualitativo/ Experimental \\
\hline A.7 & $\begin{array}{l}\text { Acesso aos serviços de saúde para o } \\
\text { diagnóstico e tratamento da tuberculose } \\
\text { entre povos indígenas do estado de } \\
\text { Rondônia, Amazônia Brasileira, entre } \\
2009 \text { e 2011: um estudo transversal. }\end{array}$ & 5 & 2019 & $\begin{array}{c}\text { Enfermagem } \\
\text { Medicina } \\
\text { Ciências Biológicas }\end{array}$ & PubMed & $\begin{array}{l}\text { Qualitativa- transversal/ } \\
\text { Experimental }\end{array}$ \\
\hline A.8 & $\begin{array}{l}\text { Tuberculose em População Indígena } \\
\text { Autodeclarada no Estado do Paraná. }\end{array}$ & 7 & 2019 & $\begin{array}{c}\text { Enfermagem } \\
\text { Ciências Biológicas } \\
\text { Medicina }\end{array}$ & BVS & Quantitativo/ Documental \\
\hline A.9 & $\begin{array}{l}\text { Desempenho de testes para o diagnóstico } \\
\text { de tuberculose pulmonar em populações } \\
\text { indígenas no Brasil: a contribuição do } \\
\text { Teste Rápido Molecular }\end{array}$ & 6 & 2019 & $\begin{array}{c}\text { Enfermagem } \\
\text { Farmácia Bioquímica } \\
\text { História } \\
\text { Ciências Biológicas } \\
\text { Medicina }\end{array}$ & BVS & Quantitativa/ experimental \\
\hline
\end{tabular}

Fonte: Autores (2021).

Nota-se que a enfermagem se destaca no que se refere a área de publicação $(58,4 \%)$, seguida pela área médica $(17,1 \%)$ e biólogos (7,3\%). A ampla participação dos profissionais da enfermagem na autoria dos artigos demonstra a relevância dessa categoria na resolutividade do problema. Além disso, a equipe de enfermagem está presente em todas as esferas da saúde e se encontra próxima das populações locais, sendo estes, na maioria das vezes, os únicos presentes nas unidades de saúde. Dessa forma, incentivos em estudos para a capacitação profissional de enfermeiros, no que tange ao conhecimento da TB, são necessários para a facilitação da atuação profissional e a diminuição dos casos presentes nas comunidades indígenas. 
Com relação às temáticas abordadas, a ampla maioria dos artigos cita a descontinuidade do tratamento, a alta prevalência dos casos nas regiões Centro-Oeste e Norte, as questões socioeconômicas e a dificultosa acessibilidade às unidades de saúde para a persistência dos casos de tuberculose.

Os anos com maiores números de publicações são 2019 com 5 artigos (55,5\%) e 2017 com 2 artigos (22,1\%). Os demais anos, 2018 e 2020, contam com apenas 1 artigo (11,2\%) cada. A abordagem metodológica prevalente nos artigos foi: quantitativa 6 artigos $(66,7 \%)$ e qualitativa 3 artigos $(33,3 \%)$.

\section{Discussão}

A incidência elevada de tuberculose nas populações indígenas comparada à incidência de TB nas demais populações demonstra que essa é uma problemática de saúde pública. A análise do material bibliográfico permitiu identificar questões relevantes para a prevalência elevada de tuberculose nas populações indígenas. E, dessa forma, o presente trabalho aborda os principais fatores determinantes para a incidência de tuberculose nas populações indígenas estando organizado nas seguintes classes: a) Questões socioeconômicas, b) Imprecisão diagnóstica, c) Acessibilidade aos centros de saúde, d) Epidemiologia.

\section{a) Questões socioeconômicas}

No que se refere à questão socioeconômica, a pesquisa mostrou que as populações mais vulneráveis, como é o caso da população indígena, são mais suscetíveis à TB. A doença se dissemina mais entre grupos que apresentem uma qualidade de vida carente e em que há um baixo nível de desenvolvimento econômico, o que justifica as condições de moradias precárias, saneamento básico insuficiente e a baixa escolaridade.

À vista disso, tais fatos são ratificados pela infraestrutura inadequada, que contêm um grande número de residentes em apenas uma casa, a qual, muitas vezes, dispõe de um único cômodo e sem banheiro. Da mesma forma, evidencia-se a escassez de ações sanitárias, especialmente as que viabilizem o acesso à água tratada e encanada, o que pode resultar em alto risco de contrair outras enfermidades, como diarréia e parasitoses. Ainda, comprova a baixa escolaridade, pois evidências científicas constatam que indivíduos não alfabetizados apresentam maiores possibilidades de serem afetados pela doença, além de terem pouca propensão pela procura de serviços de saúde (Zorzi et al., 2019).

Outrossim, ressalta-se que as pessoas que se autodeclaram indígenas e que não recebem nenhum benefício social, como bolsa família, possuem mais chances de adoecerem em decorrência de TB, tendo em vista que quanto maior os índices de vulnerabilidade mais esses povos ficam mais sujeitos a viverem em estado de pobreza, afetando diretamente o seu bem-estar (Paiva et al., 2019).

\section{b) Imprecisão diagnóstica}

As Leis $\mathrm{n}^{\circ} 8.080$ de 19 de setembro de 1990, e nº 9.836 de 23 de setembro de 1999 são documentos que garantem atendimento especializado à saúde de todo cidadão indígena. Conquanto, salientando o desenvolvimento dos diagnósticos de TB referentes aos povos indígenas, tal direito não tem se reverberado, dificultando a identificação da infecção e retardando ou até mesmo impossibilitando a recuperação da saúde dessa população. Diante desse cenário, é possível analisar alguns fatores que favorecem a imprecisão diagnóstica da tuberculose na população indígena (Wenczenovicz, 2018)

Assim, o processo de diagnose da TB pode ocorrer de duas formas, por meio da baciloscopia que é a mais utilizada por apresentar mais vantagens em relação ao tempo e aos gastos, mas apresenta 50\% de falha em casos suspeitos. A cultura de escarro, outra forma de diagnóstico, tem de $70 \%$ a $90 \%$ de eficácia, porém possui muitas exigências a serem feitas, como incubação, período de espera para que ocorra o crescimento de colônias necessário para que se possa diagnosticar a TB, o qual pode ser de 2 a 8 semanas (Malacarne et al., 2019a). 
É fundamental apontar fatores como a falta de acessibilidade e a situação econômica como um dos grandes impulsionadores da falta de imprecisão, pois as áreas indígenas em sua maioria não possuem locais próximos para atendimento, dificultando a sua ida voluntária em busca de exames e tratamentos e, em alguns casos, os indígenas acabam tendo de ir várias vezes para se ter o diagnóstico conclusivo, o que acaba pesando economicamente. Além disso, a falta de tecnologias necessárias, laboratórios e profissionais especializados contribuem para que tal falha esteja em constante manutenção. Outro fator que vale ser constatado é a grande diversidade de línguas e costumes, pois a população indígena é dividida entre 305 etnias e 247 idiomas. Logo, é necessário que ocorra uma adaptação entre os profissionais que irão estar com a população indígena em busca de um resultado conclusivo, desde materiais e pessoas que possam facilitar a comunicação, o que nem sempre é possível (Malacarne et al., 2019b; Zorzi et al., 2019).

Ademais, sabendo que a TB não atinge apenas jovens e adultos, a população indígena infantil possui mais dificuldades para obter um diagnóstico correto, pois as crianças, diferente dos adultos, não possuem sintomas que podem ser conclusivos para a TB. Além disso, a cooperação infantil para a realização dos exames é complicada e ainda pode-se ter um falso-negativo e resultados inconclusivos. Conclui-se então, que os estudos referentes à TB infantil ainda necessitam de aprofundamento (Viana, Codenotti, Bierrenbach, \& Basta, 2019).

\section{c) Acessibilidade aos centros de saúde}

Somado a isso, há o problema no acesso aos centros de saúde, não só na questão distância-econômica como também na linguística-cultural. No quesito deslocamento, alguns municípios dispõem de transporte e preparo adequados para locomovê-los até as unidades de saúde. No entanto, sabe-se que essa não é a realidade da grande maioria da população indígena, que muitas vezes precisa custear a locomoção por conta própria de seus doentes (já que na maioria das vezes estão desassistidos pelos órgãos de saúde pública e demais encarregados), o que acaba por deixar seus convalescidos desamparados e sem tratamento adequado pois, em suma, quase nunca dispõem do capital necessário (Gomes \& Esperidião, 2017).

Ademais, o deslocamento até a unidade de saúde nem sempre é facilitado, pois intercorrências, por conta das más qualidades das estradas durante o traslado, é algo corriqueiro. Além disso, os poucos que ainda contam com os recursos encontram dificuldades na precariedade das instalações físicas, na espera na mesma fila que a comunidade urbana e algumas vezes rural, e, agora, na barreira linguística-cultural (Gomes \& Esperidião, 2017).

Muitos profissionais não conseguem tratar com qualidade os pacientes indígenas. No geral, não estão capacitados para atender essa comunidade em específico, visto que há pouquíssima capacitação voltada para atender suas necessidades, o que muitas vezes gera um desconforto e, por consequência, um "mau" atendimento aos indígenas. A barreira linguística é um fator determinante na abordagem e para o tratamento da população indígena, pois é por intermédio da comunicação que se inicia o processo de atendimento. Além disso, as diferenças culturais: de vestimenta, calçado, alimentação, sono e até mesmo na forma de interagir com o ambiente são de suma importância na qualidade do tratamento. Os profissionais de saúde não indígenas, de uma forma geral, não são preparados para realizar a assistência a esses povos, sendo que a baixa capacitação é reflexo das dificuldades de investimento social e político e da conformação das políticas de saúde no Brasil (Gomes \& Esperidião, 2017).

\section{d) Epidemiologia}

Os dados retirados do sistema nacional de agravos de notificação (SINAN) tem o objetivo de verificar casos e incidências mais atuais e comparar com as informações de outros artigos. Assim, durante o período de 2015 a 2019, foram notificados 452.309 casos de TB no Brasil, sendo 91,1\% (n=412.390) na população não indígena e 1,1\% desses casos acometeram a população indígena $(\mathrm{n}=4.695)$. Foram excluídos 7,7\% do total $(\mathrm{n}=35.224)$ pela impossibilidade de definição da raça/cor. Estratificando a nível regional e estadual: na região norte, $96,1 \%(n=47.030)$ na população não indígena e 3,8\% 
( $\mathrm{n}=1.901)$ na população indígena, além disso, em relação ao estado Pará, 98,8\% ( $\mathrm{n}=21.724)$ para não indígena e 1,2\% ( $\mathrm{n}=401)$ para os povos autodeclarados indígenas.

Como foi possível observar, há muito mais casos na população não indígena (branca, preta, parda e amarela). Entretanto, a distribuição de pessoas nas duas populações é extremamente desigual: em que pelo censo demográfico de 2010, a população autodeclarada indígena era composta por 817.963 mil pessoas, representando $0,4 \%$ dos brasileiros e em relação à Região Norte do país, há 305.873 mil indígenas, o equivalente à $1,9 \%$ da população da região (Ferreira et al., 2020). Diante disso, se faz necessário calcular o coeficiente de incidência em que permite comparar os casos nas duas populações.

Portanto, nas comunidades indígenas em toda América Latina, principalmente nos grupos amazônicos brasileiros, foram verificadas taxas de incidências extremamente elevadas de TB, muitas vezes superior a 1.000/100.000 habitantes. De acordo com o mesmo artigo, no Brasil, o coeficiente de incidência de TB nos povos indígenas foi de 109/100.000 hab. durante os anos de 2011 a 2017. Nesse mesmo estudo, o Norte foi a segunda região do país com maior incidência com cerca de 124,9/100.000 habitantes e o estado do Pará, em sétimo lugar entre os estados com maiores taxas, com 165,5/100.00 habitantes (Ferreira, Santos, Oliveira \& Caldas, 2020).

Em outro artigo, analisou-se a taxa de incidência no Pará nos anos de 2012 a 2015 e foi encontrado taxas de 142,5/100.000 habitantes (Paiva et al., 2019). Do mesmo modo, no período de 2005 a 2013 em que na população não indígena o coeficiente foi de 46,0/100.000 hab., enquanto que para os indígenas os índices foram de 115,16/100.000 hab., aproximadamente 2,5 vezes maior (Paiva et al., 2017). Em outro estudo, realizado em indígenas com TB no Paraná, também foram verificados números elevados durante os anos estudados, ressaltando os anos de 2002 com coeficiente de 320/100 mil hab. e o ano de 2010 com 111,5 casos/100 mil habitantes (Zorzi et al., 2019).

Ressalta-se que o coeficiente de incidência foi calculado no mesmo molde que os estudos feitos pelos autores de Paiva et al. (2019), e Ferreira et al. (2020), com a intenção de comparar os índices.

Diante disso, foram encontrados coeficientes de incidência semelhantes nos artigos analisados. Houve, novamente, taxas elevadas de incidência média na Região Norte, na qual o coeficiente foi de 100,66/100.000 habitantes na população indígena, cerca de 2,2 vezes maior em relação à população não indígena, com o registro de 43,8/100.000 habitantes. No estado do Pará, os indígenas com TB tiverem o coeficiente médio de 160,25/100.000 habitantes no período analisado - a diferença alcança o nível de 3,6 vezes maior em relação à população não indígena - com maior incidência observada no ano de 2019 com 217,98/100.000 habitantes (Brasil, 2021).

Em relação à zona de residência, 81,7\% vivem em zona rural, o mesmo ocorre com indígenas no Paraná, cerca de 67,2\% de casos de TB eram em indivíduos da zona rural, os quais possuem diversas particularidades. Importante destacar que, a responsabilidade de fornecer acesso aos serviços de saúde na zona rural é da Secretaria Especial de Saúde Indígena (SEASI) (Brasil, 2002; Zorzi et al., 2019).

Portanto, esses dados corroboram com o fato da circulação do bacilo está relacionada com as condições econômicas e sociais desfavoráveis, o acesso limitado aos serviços de saúde e as especificidades das famílias indígenas. Somando-se a isso, a incapacidade do sistema de saúde em promover o diagnóstico e o tratamento em tempo oportuno, potencializa os agravos gerados pela doença e a persistência de coeficientes superiores dessa população em relação a outras (Brasil, 2002).

\section{Considerações Finais}

A análise do presente estudo permitiu concluir que as populações indígenas vivenciam situações de exclusão, invisibilidade e discriminação, o que as colocam em situações de vulnerabilidade. Os elevados índice de transmissão de tuberculose nas comunidades indígenas comparado aos níveis de transmissão nas populações em geral permite constatar que a tuberculose afeta desproporcionalmente aos indígenas e que, dessa forma, a PNASPI não está sendo efetuada integralmente. 
Além disso, o presente estudo permitiu realçar que essa problemática envolve questões multisetoriais e que a superação dos determinantes em saúde para a prevalência de tuberculose envolve a articulação e colaboração entre o Governo e as comunidades indígenas locais.

Constatou-se que a má distribuição de renda associada aos problemas de descasos sociais e sanitários estão intimamente relacionados aos casos de TB. Portanto, programas de transferência de renda e políticas públicas voltadas às necessidades dessas populações são necessárias para a superação desses agravos em saúde. Nota-se também que investimentos em ampliação do acesso ao Sistema Público de Saúde; modernização e disponibilização de medicamentos e procedimentos diagnósticos; acessibilidade aos serviços de saúde dentro dos distritos indígenas e a capacitação dos profissionais e populações locais para a atuação nos serviços de saúde são fundamentais para o correto manuseio, notificação e prevenção dos casos de tuberculose.

A atualização epidemiológica sobre a incidência de tuberculose nas populações indígenas também se mostra como uma contribuição fundamental deste trabalho. E esperamos que esforços contínuos para o controle e a diminuição dos casos de tuberculose nas populações indígenas possam ser associados a metas tangíveis e abrangentes nos seus diversos fatores determinantes. Assim, será possível garantir aos povos indígenas o acesso à atenção integral à saúde, conforme preconizado pelos princípios e diretrizes do SUS.

Além disso, evidenciamos que existem poucos estudos com esta temática, sendo visível pela dificuldade dos autores em encontrar publicações que abordem a epidemiologia como aspecto central. Desse modo, sugerimos para os futuros trabalhos desenvolvidos, a constante busca de conhecimento técnico e científico acerca do assunto em questão, tendo em vista a alta prevalência e incidência de TB nas populações indígenas e a importância da compreensão da situação epidemiológica para o processo de implementação de políticas públicas.

\section{Referências}

Brasil. Ministério da Saúde. (2002). Política Nacional de Atenção à Saúde dos Povos Indígenas (2a edição). Fundação Nacional de Saúde, aprovada pela Portaria do Ministério da Saúde no 254, de 31 de janeiro de 2002.

Brasil. Ministério da Saúde. (2021). Sistema De Informações De Agravos De Notificação - Sinan. Secretaria de Vigilância em Saúde, Departamento de Vigilância Epidemiológica.

Brasil. Ministério da Saúde. (2004). Programa Nacional de Controle da Tuberculose. Secretaria de Vigilância em Saúde, Departamento de Vigilância Epidemiológica.

Castro, D. B. de, Maciel, E. M. G. de S., Sadahiro, M., Pinto, R. C., Albuquerque, B. C. de, \& Braga, J. U. (2018). Tuberculosis incidence inequalities and it's social determinants in Manaus from 2007 to 2016. International Journal for Equity in Health, 17(1), 187.

Cortez, A. O., Melo, A. C. de, Neves, L. de O., Resende, K. A., \& Camargo, P. (2021). Tuberculose no Brasil: um país, múltiplas realidades. Jornal Brasileiro de Pneumologia, 47(2), e20200119.

Crossetti, M. da G. O. (2012). Revisão integrativa de pesquisa na enfermagem o rigor cientifico que lhe é exigido. Revista Gaúcha de Enfermagem, 33(2).

Ercole, F. F., Melo, L. S. de, \& Alcoforado, C. L. G. C. (2014). Revisão integrativa versus revisão sistemática. Reme: Revista Mineira de Enfermagem, 18(1), $9-11$.

Ferreira, T. F., Santos, A. M. dos, Oliveira, B. L. C. A. de, \& Caldas, A. de J. M. (2020). Tendência da tuberculose em indígenas no Brasil no período de 2011 2017. Ciência \& Saúde Coletiva, 25(10), 3745-3752.

Gomes, S. C., \& Esperidião, M. A. (2017). Acesso dos usuários indígenas aos serviços de saúde de Cuiabá, Mato Grosso, Brasil. Cadernos de Saúde Pública, $33(5), \mathrm{e} 00132215$.

Malacarne, J. (2013). Tuberculose na população indígena de Rondônia: caracterização do acesso aos serviços de saúde e diagnóstico situacional entre os Wari da aldeia Igarapé Ribeirão (Dissertação de mestrado). Escola Nacional de Saúde Pública Sergio Arouca, Rio de Janeiro, RJ, Brasil.

Malacarne, J., Heirich, A. S., Cunha, E. A. T., Kolte, I. V., Souza-Santos, R., \& Basta, P. C. (2019a). Desempenho de testes para o diagnóstico de tuberculose pulmonar em populações indígenas no Brasil: a contribuição do Teste Rápido Molecular. Jornal Brasileiro de Pneumologia, $45(2)$, e20180185.

Malacarne, J., Gava, C., Escobar, A. L., Souza-Santos, R., \& Basta, P. C. (2019b). Acesso aos serviços de saúde para o diagnóstico e tratamento da tuberculose entre povos indígenas do estado de Rondônia, Amazônia Brasileira, entre 2009 e 2011: um estudo transversal. Epidemiologia e Serviços de Saúde, 28(3), e2018231. 
Research, Society and Development, v. 10, n. 6, e8110615417, 2021

(CC BY 4.0) | ISSN 2525-3409 | DOI: http://dx.doi.org/10.33448/rsd-v10i6.15417

Paiva, B. L., Azeredo, J. Q., Nogueira, L. M. V., Santos, B. de O., Rodrigues, I. L. A., \& Santos, M. N. de A. (2017). Distribuição espacial de tuberculose nas populações indígenas e não indígenas do estado do Pará, Brasil, 2005-2013. Escola Anna Nery, 21(4), e20170135.

Paiva, B. L., Nogueira, L. M. V., Rorigues, I. L. A., Basta, P. C., Ferreira, Â. M. R., \& Caldas, S. P. (2019). Modelo preditivo de determinantes socioeconômicos da tuberculose em população indígena do Estado do Pará, Brasil. Cogitare Enfermagem, 24, e64835.

Polit, D. F., \& Beck, C. T. (2019). Fundamentos de pesquisa em enfermagem: avaliação de evidências para a prática da enfermagem. Artmed.

Sandes L. F. F., Freitas D. A., Souza M. F. N. S de, \& Leite K. B. de S. (2018). Atenção primária à saúde de indígenas sul-americanos: revisão integrativa da literatura. Revista Panamericana de Salud Pública, 42, e163.

Souza, T. J. de. (2017). Processo saúde-doença em indígenas: reflexões sobre a ocorrência de tuberculose no Brasil. Revista Eletrônica do Univag - Connection line, 17, 153-162.

Viana, P. V. de S., Codenotti, S. B., Bierrenbach, A. L., \& Basta, P. C. (2019). Tuberculose entre crianças e adolescentes indígenas no Brasil: fatores associados ao óbito e ao abandono do tratamento. Cadernos de Saúde Pública, 35(Suppl. 3), e00074218.

Wenczenovicz, T. J. (2018). Saúde Indígena: Reflexões Contemporâneas. Revista Cadernos Ibero-Americanos de Direito Sanitário, 7(1), 63-82.

Zorzi, S. S., Campos, R. B., Quadros, S. R. de Zilly, A., Palha, P. F., Gabardo, B. M. A., \& Silva-Sobrinho, R. A. (2019). Tuberculose em população indígena autodeclarada no Estado do Paraná. Revista Brasileira De Ciências Da Saúde, 23(1). 